COMPUTATIONAL MECHANICS

New Trends and Applications

S. Idelsohn, E. Oñate and E. Dvorkin (Eds.)

(c) CIMNE, Barcelona, Spain 1998

\title{
RECENT DEVELOPMENT OF DUAL BEM IN ACOUSTIC PROBLEMS
}

\author{
J. T. Chen \\ Department of Harbor and River Engineering, National Ocean University, Keelung, \\ Taiwan \\ P.O. Box 7-59, Keelung, Taiwan \\ e-mail:b0209@ntou66.ntou.edu.tw
}

\section{Key words:Dual integral formulation, Dual boundary element method, Acous- tic problem, Degenerate boundary, Multiple reciprocity method}

\begin{abstract}
In this lecture, recent development of the dual BEM in acoustic problem is presented. The role of hypersingular integral equation in the dual BEM for the problems with degenerate boundary is examined. First, the dual integral formulation is proposed. Based on the formulation, we develop four methods - the dual complex-valued BEM, the real part of dual BEM, the dual real-valued $M R M$, and the complete complex-valued MRM. After constructing the dual MRM, spurious eigenvalues in the conventional MRM can be filtered out. It is also found that the complete MRM with infinite terms of series is equivalent to the dual complex-valued BEM if the constant potential in the zeroth-order fundamental solution is chosen to be an appropriate complex value. The dual formulation can be applied to solve acoustic problems with degenerate boundaries. An illustrative example of acoustic frequencies of a cavity with an incomplete partition are determined by the four methods. The results are compared with those of FEM and experiment. Good agreement is made.
\end{abstract}




\section{INTRODUCTION}

The boundary element method, sometimes referred to as the boundary integral equation method, is now establishing a position as a natural alternative to the FEM in many fields of engineering. The dual boundary element method, or so-called the dual boundary integral equation method developed by Chen and Hong, is particularly suited for problems with degenerate boundary. Mathematically speaking, the hypersingular integral equation was first formulated by Hadamard [1] to treat the cylindrical wave equation by spherical means of descent. In the meantime, Mangler derived the same mathematical form in solving the thin airfoil problem [2]. The improper integral was then defined by Tuck [3] as the "Hadamard principal value". In aerodynamics, it was termed the "Mangler's principal value" $[2,4]$. Such a nonintegrable integral naturally arises in the dual integral formulation especially for problems with a degenerate boundary, e.g., crack problems in elasticity $[5,6,7,8,9,10]$, heat flow through a baffle [11], Darcy flow around a cutoff wall $[12,13]$, the aerodynamic problem of a thin airfoil [4] and acoustic waves impinging on a screen $[14,15]$. The dual formulation also plays an important role in some other problems, e.g., the corner problem [16], adaptive BEM [17], and the exterior problem [18]. A general application of the hypersingular integral equation in mechanics was discussed in [19], and a review lecture on recent development of dual BEM was presented in [20]. Combining the conventional integral equation, e.g., the Green's Identity or Somigliana Identity, with the hypersingular integral equation, we call the two equations "dual integral equations" due to the symmetry and transpose symmetry properties of the kernels. $[21,22,23]$. From the above point of view, the definition of the dual integral equations is quite different from the conventional one used in crack elastodynamics by Buecker [24]. The dual equations in the present paper are independent with respect to each other for the undetermined coefficients of the complementary solution. The dual integral equations defined by Buecker resulted from the same equation but by collocating different points. The present formulation totally has four kernel functions, which make possible a unified theory encompassing different schemes, various derivations and interpretations. For elasticity, a detailed derivation can be found in [5]. The singularity order of hypersingularity for the kernel in the normal derivative of the double layer potential is stronger than that of the Cauchy type kernel by one. The paradox of the nonintegrable kernel is introduced due to the illegal change of the integral and trace operators from the point of view of the dual integral formulation [21]. In order to ensure a finite value, the Leibnitz's rule should

be considered as the derivative of C.P.V. so that the boundary term $\frac{2}{\epsilon}$ can be included to compensate for the minus infinity. In the literature, many researchers have paid attention to regularization techniques [25] for hypersingularity and nearly hypersingular integrals. Therefore, the value for the finite part can be determined by means of regularization techniques. Based on the theory of dual integral equations, the dual boundary element method can be implemented $[9,10]$. The dual integral representation for the Laplace equation was proposed in [22] and a general program, BEPO2D, was developed [21]. In the same way, the acoustic problem with a degenerate boundary also requires the dual integral formulation. In the literature, a large number of papers have focused on the nonphysical 
solution for the exterior problem of the Helmholtz equation by using the integral equation method. Burton and Miller [27] first combined the dual integral equations to deal with fictitious eigenvalues. Furthermore, the conventional multiple reciprocity method(MRM) also encounters spurious eigenvalues for the interior problem of the Helmholtz equation [28]. Both cases, the exterior problem by BEM and the interior problem by MRM, have problems with nonuniqueness for the solution. However, for the interior problem with a degenerate boundary, conventional BEM also results in a singular system, and the problem of nonuniqueness also occurs. Terai [14] and $\mathrm{Wu}$ et al. [15] solved the three-dimensional acoustic problem with a screen by using the dual integral formulation. A general program, DUALHAK, was developed to solve the acoustic frequencies and modes for a cavity with an incomplete partition in [31, 32].

In this paper, the dual integral equations was constructed for acoustic problems with degenerate boundaries. Based on the dual formulations, four methods - the dual complex-valued BEM, the dual real-valued MRM, the real part of the dual BEM and complete complex-valued MRM, were proposed. An illustrative example for the acoustic frequencies of a cavity with an incomplete partition was demonstrated to show the validity of the four methods. Results were compared with those of FEM by Petyt [33, 34] and ABAQUS. Also, the experimental data by Petyt [33, 34] are available.

\section{DUAL INTEGRAL FORMULATION FOR AN ACOUSTIC PROBLEM WITH A DEGENERATE BOUNDARY}

Consider an acoustic problem which has the following governing equation:

$$
\nabla^{2} \phi(\mathbf{x})+k^{2} \phi(\mathbf{x})=0, \mathbf{x} \in D,
$$

where $D$ is the domain of interest, $\mathbf{x}$ is the domain point, $\phi$ is the acoustic pressure and $k$ is the wave number defined by the angular frequency divided by the sound speed. The homogeneous boundary conditions are shown as follows:

$$
\begin{gathered}
\phi(\mathbf{x})=0, \mathbf{x} \in B_{1} \\
\frac{\partial \phi(\mathbf{x})}{\partial n_{\mathbf{x}}}=0, \mathbf{x} \in B_{2},
\end{gathered}
$$

where $B_{1}$ is the essential boundary in which the acoustic pressure is prescribed, $B_{2}$ is the natural boundary where the normal derivative of the acoustic pressure in the $n_{x}$ direction is specified, and $B_{1}$ and $B_{2}$ construct the whole boundary of the domain $D$.

The first equation of the dual boundary integral equations for the domain point can be derived from Green's third identity :

$$
2 \pi \phi(\mathbf{x})=\int_{B} T(\mathbf{s}, \mathbf{x}) \phi(\mathbf{s}) d B(\mathbf{s})-\int_{B} U(\mathbf{s}, \mathbf{x}) \frac{\partial \phi(\mathbf{s})}{\partial n_{\mathbf{s}}} d B(\mathbf{s}), \mathbf{x} \in D,
$$

where $T(\mathbf{s}, \mathbf{x})$ is defined by

$$
T(\mathbf{s}, \mathbf{x}) \equiv \frac{\partial U(\mathbf{s}, \mathbf{x})}{\partial n_{\mathbf{s}}}
$$


in which $n_{\mathbf{s}}$ represents the outnormal direction at point $\mathbf{s}$ on the boundary and $U(\mathbf{s}, \mathbf{x})$ is the fundamental solution which satisfies

$$
\nabla^{2} U(\mathbf{x}, \mathbf{s})+k^{2} U(\mathbf{x}, \mathbf{s})=\delta(\mathbf{x}-\mathbf{s}), \mathbf{x} \in D
$$

where $\delta(\mathbf{x}-\mathbf{s})$ is the Dirac delta function. After taking the normal derivative with respect to Eq.(4), the second equation of the dual boundary integral equations for the domain point can be derived:

$$
2 \pi \frac{\partial \phi(\mathbf{x})}{\partial n_{\mathbf{x}}}=\int_{B} M(\mathbf{s}, \mathbf{x}) \phi(\mathbf{s}) d B(\mathbf{s})-\int_{B} L(\mathbf{s}, \mathbf{x}) \frac{\partial \phi(\mathbf{s})}{\partial n_{\mathbf{s}}} d B(\mathbf{s}), \mathbf{x} \in D
$$

where

$$
\begin{aligned}
L(\mathbf{s}, \mathbf{x}) & \equiv \frac{\partial U(\mathbf{s}, \mathbf{x})}{\partial n_{\mathbf{x}}} \\
M(\mathbf{s}, \mathbf{x}) & \equiv \frac{\partial^{2} U(\mathbf{s}, \mathbf{x})}{\partial n_{\mathbf{x}} \partial n_{\mathbf{s}}}
\end{aligned}
$$

in which $n_{\mathbf{x}}$ represents the outnormal direction at point $\mathbf{x}$. The explicit forms of the four kernel functions will be elaborated later on. By moving the field point $x$ in Eqs.(4) and (7) to the smooth boundary, the dual boundary integral equations for the boundary point can be obtained as follows:

$$
\begin{gathered}
\pi \phi(\mathbf{x})=C . P . V . \int_{B} T(\mathbf{s}, \mathbf{x}) \phi(\mathbf{s}) d B(\mathbf{s})-R . P . V . \int_{B} U(\mathbf{s}, \mathbf{x}) \frac{\partial \phi(\mathbf{s})}{\partial n_{\mathbf{s}}} d B(\mathbf{s}), \mathbf{x} \in B, \\
\pi \frac{\partial \phi(\mathbf{x})}{\partial n_{\mathbf{x}}}=H . P . V . \int_{B} M(\mathbf{s}, \mathbf{x}) \phi(s) d B(\mathbf{s})-C . P . V . \int_{B} L(\mathbf{s}, \mathbf{x}) \frac{\partial \phi(\mathbf{s})}{\partial n_{\mathbf{s}}} d B(\mathbf{s}), \mathbf{x} \in B,
\end{gathered}
$$

where R.P.V. is the Riemann principal value, C.P.V. is the Cauchy principal value and H.P.V. is the Hadamard (Mangler) principal value.

It must be noted that Eq.(11) can be derived simply by applying the normal derivative operator to Eq.(10). Differentiation of the Cauchy principal value should be carried out carefully using Leibnitz's rule. The commutative property provides us with two alternatives for calculating the Hadamard principal value in the same way used for crack problems [5]. For the problem including a normal boundary $S$ and degenerate boundary $C^{+}+C^{-}$, i.e., $B=S+C^{+}+C^{-}$, Eqs.(10) and (11) can be reformulated as follows: For $\mathbf{x} \in \mathrm{S}$, Eqs.(10) and (11) become

$$
\begin{aligned}
\pi \phi(\mathbf{x})= & C . P . V . \int_{S} T(\mathbf{s}, \mathbf{x}) \phi(\mathbf{s}) d B(\mathbf{s})-R . P . V . \int_{S} U(\mathbf{s}, \mathbf{x}) \frac{\partial \phi(\mathbf{s})}{\partial n_{\mathbf{s}}} d B(\mathbf{s}) \\
& +\int_{C^{+}} T(\mathbf{s}, \mathbf{x}) \Delta \phi(\mathbf{s}) d B(\mathbf{s})-\int_{C^{+}} U(\mathbf{s}, \mathbf{x}) \Sigma \frac{\partial \phi(\mathbf{s})}{\partial n_{\mathbf{s}}} d B(\mathbf{s})
\end{aligned}
$$




$$
\begin{aligned}
\pi \frac{\partial \phi(\mathbf{x})}{\partial n_{\mathbf{x}}}= & H . P . V . \int_{S} M(\mathbf{s}, \mathbf{x}) \phi(\mathbf{s}) d B(\mathbf{s})-C . P . V . \int_{S} L(\mathbf{s}, \mathbf{x}) \frac{\partial \phi(\mathbf{s})}{\partial n_{\mathbf{s}}} d B(\mathbf{s}) \\
& +\int_{C^{+}} M(\mathbf{s}, \mathbf{x}) \Delta \phi(\mathbf{s}) d B(\mathbf{s})-\int_{C^{+}} L(\mathbf{s}, \mathbf{x}) \Sigma \frac{\partial \phi(\mathbf{s})}{\partial n_{\mathbf{s}}} d B(\mathbf{s}),
\end{aligned}
$$

where

$$
\begin{aligned}
\Delta \phi(\mathbf{s}) & =\phi\left(\mathbf{s}^{+}\right)-\phi\left(\mathbf{s}^{-}\right) \\
\Sigma \frac{\partial \phi}{\partial n}(\mathbf{s}) & =\frac{\partial \phi}{\partial n}\left(\mathbf{s}^{+}\right)+\frac{\partial \phi}{\partial n}\left(\mathbf{s}^{-}\right) .
\end{aligned}
$$

For $\mathbf{x} \in C^{+}$, Eqs.(10) and (11) reduce to

$$
\begin{aligned}
\pi \Sigma \phi(\mathbf{x})= & C . P . V \cdot \int_{C^{+}} T(\mathbf{s}, \mathbf{x}) \Delta \phi(\mathbf{s}) d B(\mathbf{s})-R . P . V \cdot \int_{C^{+}} U(\mathbf{s}, \mathbf{x}) \Sigma \frac{\partial \phi(\mathbf{s})}{\partial n_{\mathbf{s}}} d B(\mathbf{s}) \\
& +\int_{S} T(\mathbf{s}, \mathbf{x}) \phi(\mathbf{s}) d B(\mathbf{s})-\int_{S} U(\mathbf{s}, \mathbf{x}) \frac{\partial \phi(\mathbf{s})}{\partial n_{\mathbf{s}}} d B(\mathbf{s}), \\
\pi \Delta \frac{\partial \phi(\mathbf{x})}{\partial n_{\mathbf{x}}}= & H . P . V \cdot \int_{C^{+}} M(\mathbf{s}, \mathbf{x}) \Delta \phi(\mathbf{s}) d B(\mathbf{s})-C . P . V \cdot \int_{C^{+}} L(\mathbf{s}, \mathbf{x}) \Sigma \frac{\partial \phi(\mathbf{s})}{\partial n_{\mathbf{s}}} d B(\mathbf{s}) \\
& +\int_{S} M(\mathbf{s}, \mathbf{x}) \phi(\mathbf{s}) d B(\mathbf{s})-\int_{S} L(\mathbf{s}, \mathbf{x}) \frac{\partial \phi(\mathbf{s})}{\partial n_{\mathbf{s}}} d B(\mathbf{s}),
\end{aligned}
$$

where

$$
\begin{aligned}
\Sigma \phi(\mathbf{x}) & =\phi\left(\mathbf{x}^{+}\right)+\phi\left(\mathbf{x}^{-}\right) \\
\Delta \frac{\partial \phi}{\partial n}(\mathbf{x}) & =\frac{\partial \phi}{\partial n}\left(\mathbf{x}^{+}\right)-\frac{\partial \phi}{\partial n}\left(\mathbf{x}^{-}\right) .
\end{aligned}
$$

Eqs.(14), (15), (18) and (19) indicate that the unknowns on the degenerate boundary double, and that the additional hypersingular integral equation, Eq.(17), is correspondingly necessary; i.e., the dual boundary integral equations can provide us with sufficient constraint relations for the doubled boundary unknowns on the degenerate boundary.

Based on the dual integral formulation, four methods can be treated as special cases and are discussed in the following subsections.

\subsection{Dual complex-valued BEM}

For simplicity, a two-dimensional case is considered here. The closed forms of the four kernels in the dual complex-valued BEM are shown below:

$$
\begin{aligned}
U(\mathbf{s}, \mathbf{x}) & =\frac{-i \pi H_{0}^{(1)}(k r)}{2} \\
T(\mathbf{s}, \mathbf{x}) & =\frac{-i k \pi}{2} H_{1}^{(1)}(k r) \frac{y_{i} n_{i}}{r}
\end{aligned}
$$




$$
\begin{aligned}
L(\mathbf{s}, \mathbf{x}) & =\frac{i k \pi}{2} H_{1}^{(1)}(k r) \frac{y_{i} \bar{n}_{i}}{r} \\
M(\mathbf{s}, \mathbf{x}) & =\frac{-i k \pi}{2}\left\{-k \frac{H_{2}^{(1)}(k r)}{r^{2}} y_{i} y_{j} n_{i} \bar{n}_{j}+\frac{H_{1}^{(1)}(k r)}{r} n_{i} \bar{n}_{i}\right\}
\end{aligned}
$$

where $i^{2}=-1, r=|\mathbf{x}-\mathbf{s}|, H_{n}^{(1)}(k r)$ denotes the first kind Hankel function with order $n$, and $n_{i}$ and $\bar{n}_{i}$ denote the ith components of the normal vectors at $\mathbf{s}$ and $\mathbf{x}$, respectively.

\subsection{Dual real-valued MRM}

By employing the conventional MRM [26, 29, 36], we have the two kernels

$$
\begin{aligned}
& U(\mathbf{s}, \mathbf{x})=U^{0}(\mathbf{s}, \mathbf{x})-k^{2} U^{1}(\mathbf{s}, \mathbf{x})+k^{4} U^{2}(\mathbf{s}, \mathbf{x})+\cdots \\
& T(\mathbf{s}, \mathbf{x})=T^{0}(\mathbf{s}, \mathbf{x})-k^{2} T^{1}(\mathbf{s}, \mathbf{x})+k^{4} T^{2}(\mathbf{s}, \mathbf{x})+\cdots
\end{aligned}
$$

where the explicit forms of $U^{j}(\mathbf{s}, \mathbf{x})$ and $T^{j}(\mathbf{s}, \mathbf{x})$ will be introduced later. In order to filter out the spurious modes, the dual MRM proposed the hypersingular integral equation with the following two kernels

$$
\begin{aligned}
L(\mathbf{s}, \mathbf{x}) & =L^{0}(\mathbf{s}, \mathbf{x})-k^{2} L^{1}(\mathbf{s}, \mathbf{x})+k^{4} L^{2}(\mathbf{s}, \mathbf{x})+\cdots \\
M(\mathbf{s}, \mathbf{x}) & =M^{0}(\mathbf{s}, \mathbf{x})-k^{2} M^{1}(\mathbf{s}, \mathbf{x})+k^{4} M^{2}(\mathbf{s}, \mathbf{x})+\cdots,
\end{aligned}
$$

in which

$$
\begin{aligned}
L^{j}(\mathbf{s}, \mathbf{x}) & =\frac{\partial U^{j}(\mathbf{s}, \mathbf{x})}{\partial n_{\mathbf{x}}}, j=0,1,2, \cdots \\
M^{j}(\mathbf{s}, \mathbf{x}) & =\frac{\partial^{2} U^{j}(\mathbf{s}, \mathbf{x})}{\partial n_{\mathbf{x}} \partial n_{\mathbf{s}}}, j=0,1,2, \cdots
\end{aligned}
$$

The explicit forms of the jth terms in the four kernels by using the dual real-valued MRM are

$$
\begin{aligned}
U^{j}(\mathbf{s}, \mathbf{x})= & r^{2 j} \ln (r) A(j)-r^{2 j} B(j) \\
T^{j}(\mathbf{s}, \mathbf{x})= & -\left[(2 j \ln (r)+1) r^{2 j-2} y_{i} n_{i}\right] A(j)+\left[2 j r^{2 j-2} y_{i} n_{i}\right] B(j) \\
L^{j}(\mathbf{s}, \mathbf{x})= & +\left[(2 j \ln (r)+1) r^{2 j-2} y_{i} \bar{n}_{i}\right] A(j)-\left[2 j r^{2 j-2} y_{i} \bar{n}_{i}\right] B(j) \\
M^{j}(\mathbf{s}, \mathbf{x})= & -\left[(4 j(j-1) \ln (r)+4 j-2) r^{2 j-4} y_{i} n_{i} y_{k} \bar{n}_{k}\right] A(j) \\
& -\left[(2 j \ln (r)+1) r^{2 j-2} n_{i} \bar{n}_{i}\right] A(j) \\
& +\left[4 j(j-1) r^{2 j-4} y_{i} n_{i} y_{k} \bar{n}_{k}\right] B(j) \\
& +\left[2 j r^{2 j-2} n_{i} \bar{n}_{i}\right] B(j) .
\end{aligned}
$$

where $A(j)$ and $B(j)$ in Eq.(30) can be found in $[29,36]$. After constructing the hypersingular integral equation, the dual MRM can filter out the spurious modes and frequencies. Nevertheless, the dual MRM can not solve the problems with impedance boundary conditions since the information on the imaginary part is lost. Also, this is the reason why 
the conventional MRM with only UT equation can not solve for the exterior problems since the method can not satisfy the radiation condition. The applications of dual MRM to the vibration problems of a rod and a beam can be found in [26, 37].

\subsection{Real part of dual BEM}

According to the findings by Yeih et al. [28] and Kamiya et al. [35], the series forms of the kernels in the dual real-valued MRM are no more than the real parts of the closedform kernels in the dual complex-valued BEM. The closed-form kernels for the real part of dual BEM are shown below:

$$
\begin{aligned}
U(\mathbf{s}, \mathbf{x}) & =\operatorname{Re}\left\{\frac{-i \pi H_{0}^{(1)}(k r)}{2}\right\} \\
T(\mathbf{s}, \mathbf{x}) & =\operatorname{Re}\left\{\frac{-i k \pi}{2} H_{1}^{(1)}(k r) \frac{y_{i} n_{i}}{r}\right\} \\
L(\mathbf{s}, \mathbf{x}) & =\operatorname{Re}\left\{\frac{i k \pi}{2} H_{1}^{(1)}(k r) \frac{y_{i} \bar{n}_{i}}{r}\right\} \\
M(\mathbf{s}, \mathbf{x}) & =\operatorname{Re}\left\{\frac{-i k \pi}{2}\left\{-k \frac{H_{2}^{(1)}(k r)}{r^{2}} y_{i} y_{j} n_{i} \bar{n}_{j}+\frac{H_{1}^{(1)}(k r)}{r} n_{i} \bar{n}_{i}\right\}\right\}
\end{aligned}
$$

where $R e$ denotes the real part. In the same way, this method has the problem of spurious modes as the real-valued MRM does if the singular integral equation (UT equation) is only used. Also, this method as well as the dual real-valued MRM, can not treat the exterior problems and interior problems with impedance boundary conditions. The main advantage of this method is that it can solve problems in the real domain without the lengthy derivation of the series kernels in MRM.

\subsection{Complete complex-valued MRM}

Recently, Yeih et al. [28] proposed a complete MRM which can recover the information of the imaginary part. The main difference between the complete MRM and dual complex-valued BEM is the kernel representation. The kernels in the complete MRM are the same as those of dual complex-valued BEM after series expansion. The series forms can be represented by

$$
\begin{aligned}
U(\mathbf{s}, \mathbf{x}) & =\sum_{j=0}^{\infty}\left(-k^{2}\right)^{j} U_{j}(\mathbf{s}, \mathbf{x}) \\
T(\mathbf{s}, \mathbf{x}) & =\frac{\partial U(\mathbf{s}, \mathbf{x})}{\partial n_{\mathbf{s}}} \\
L(\mathbf{s}, \mathbf{x}) & =\frac{\partial U(\mathbf{s}, \mathbf{x})}{\partial n_{\mathbf{x}}} \\
M(\mathbf{s}, \mathbf{x}) & =\frac{\partial^{2} U(\mathbf{s}, \mathbf{x})}{\partial n_{\mathbf{x}} \partial n_{\mathbf{s}}}
\end{aligned}
$$


where

$$
\begin{gathered}
U_{0}(\mathbf{s}, \mathbf{x})=\ln (r)+\left(\gamma+\ln \left(\frac{k}{2}\right)\right)-\frac{\pi}{2 i}, \quad j=0 \\
U_{j}(\mathbf{s}, \mathbf{x})=4 F_{j}\left(\ln (r)-S_{j}\right)+F_{j}\left(\gamma+\ln \left(\frac{k}{2}\right)\right)+\frac{\pi i}{2} F_{j}, \quad j=1,2,3, \cdots
\end{gathered}
$$

in which

$$
\begin{gathered}
\gamma=\lim _{j \rightarrow \infty}\left(\sum_{l=1}^{j} \frac{1}{l}-\ln (j)\right) \\
F_{j}=\frac{r^{2 j}}{(j !)^{2} 4^{j}} \\
S_{j}=\sum_{l=1}^{j} \frac{1}{l}
\end{gathered}
$$

It is interesting to find that the difference between $U^{0}(\mathbf{s}, \mathbf{x})$ in Eq.(30) for $j=0$ and $U_{0}(\mathbf{x}, \mathbf{s})$ in Eq.(42) is only a complex constant which can make the kernel in the complete MRM satisfy the radiation condition. Also, the kernel functions of the complete MRM in Eq.(38) with infinite terms can be proved to be equal to that of the dual complex-valued BEM in Eq.(20) after series expansion.

\section{AN ILLUSTRATIVE EXAMPLE}

To demonstrate the validity of the four methods using dual formulation, an example given by Petyt $[33,34]$ is considered. A two-dimensional cavity enclosed by rigid walls is shown in Fig.1. The cavity is a rectangle, $236 \mathrm{~mm}$ long and $113 \mathrm{~mm}$ high, and contains a rigid partition located halfway along the longer side of the cavity. The thickness of the partition is modeled as zero thickness; i.e., the boundary of partition is degenerate. The partition extends from one side of the cavity halfway across to the other wall. The cavity is filled with an acoustic fluid whose density is $1.0 \mathrm{~kg} / \mathrm{m}^{3}$ and whose bulk modulus is $0.1183 \mathrm{MPa}$. The former five acoustic frequencies given in Table 1 were solved using the four methods, and the results were compared with those of ABAQUS program [29, 30] and FEM by Petyt [33, 34]. Two types of elements in the ABAQUS program, AC2D4 and AC2D8, were considered. Although no mesh convergence studies have been performed, the close agreement between the acoustic frequencies and the acoustic modes of the present results in coarse mesh and those given by Petyt et al. suggests that the mesh is adequate. For the first mode, the present results are also in better agreement with the experimental data obtained by Petyt than they are with the data obtained using other numerical methods.

\section{CONCLUSIONS}

The general formulation of the dual integral equations of the boundary value problem for the two-dimensional Helmholtz equation with a degenerate boundary has been derived in this paper. Four methods based on the dual formulation, the dual complex-valued BEM, 
the dual real-valued MRM, the real part of dual BEM and the complete complex-valued MRM, have been proposed. An illustrative example has been successfully solved using the four methods, and the results have compared well with those obtained using other numerical methods and experiments.

\section{ACKNOWLEDGEMENT}

Financial support from the National Science Council, Grant No. NSC-86-2211-E-019006, for National Taiwan Ocean University is gratefully acknowledged. The author also wishes to acknowledge Prof. H.-K. Hong and Dr. W. Yeih for their helpful discussions.

\section{References}

[1] J. Hadamard, Lectures on Cauchy's problem in linear partial differential equations, Dover, 1952.

[2] K. W. Mangler, Improper integrals in theoretical aerodynamics, RAE Report, No.2424, 1951.

[3] E. O. Tuck, Application and solution of Cauchy singular integral equations, in The Application and Numerical Solution of Integral Equations, edited by R. S. Anderson et al., Sijthoff and Noordhoff, 1980.

[4] C. S. Wang, S. Chu and J. T. Chen, Boundary element method for predicting store airloads during its carriage and separation procedures, Computational Engineering with Boundary Elements, Vol.1, Fluid and Potential Problems, edited by Grilli et al., CMP, 1990.

[5] H.-K. Hong, and J. T. Chen, Derivations of integral equations in elasticity, Journal of Engineering Mechanics Division, ASCE, 114(6), Em5, 1028-1044 (1988).

[6] J. T. Chen, On Hadamard principal value and boundary integral formulation of fracture mechanics, Master Thesis, Institute of Applied Mechanics, National Taiwan University, Taipei, Taiwan, 1986.

[7] H.-K. Hong and J. T. Chen, Generality and special cases of dual integral equations of dual integral equations of elasticity, J. CSME, 9(1), 1-19 (1989).

[8] J. T. Chen and H.-K. Hong, On Hadamard principal value and its application to crack problems through BEM, Proceeding of The 11th National Conference on Theo. and Appl. Mech., Taiwan, 1987.

[9] A. Portela, M. H. Aliabadi and D. P. Rooke, The dual boundary element method : effective implementation for crack problems, Int. J. Num. Meth. Engng., 33, 12691287 (1992). 
[10] Y. Mi, and M. H. Aliabadi, Dual boundary element method for three-dimensional fracture mechanics analysis, Eng. Anal. with Bound. Elem., 10, 161-171 (1992).

[11] J. T. Chen, and H.-K. Hong, Application of integral equations with superstrong singularity to steady state heat conduction, Thermochimica Acta, 135, 133-138 (1988).

[12] J. T. Chen and H.-K. Hong, Singularity in Darcy flow around a cutoff wall, in Advances in Boundary Elements, Vol.2, Field and Flow Solution, edited by C.A. Brebbia and J.J. Connor, 1989, pp.15-27.

[13] L. J. Gray, Boundary element method for regions with thin internal cavities, Engineering Analysis with Boundary Elements, 6(4), 180-184 (1989).

[14] T. Terai, On calculation of sound field around three dimensional objects by integral equation method, J. Sound and Vibration, 69, 71-100 (1980).

[15] T. W. Wu and G. C. Wan, Numerical modelling of acoustic radiation and scattering from thin bodies using a Cauchy principal integral equation, J. Acoust. Soc. Amer., 92, 2900-2906 (1992).

[16] J. T. Chen and H.-K. Hong, Dual boundary integral equations at a corner using contour approach around singularity, Advances in Engineering Softwares, 21(3), 169-178 (1994).

[17] M. T. Liang, J. T. Chen and S. S. Yang, Error estimation for boundary element method, Submitted, 1998.

[18] J. T. Chen, M. T. Liang and S. S. Yang, Dual boundary integral equations for exterior problems, Engineering Analysis with Boundary Elements, 16, 33-340 (1995).

[19] P. A. Martin, F. J. Rizzo and I. R. Gonsalves, On hypersingular integral equations for certain problems in mechanics, Mech. Res. Commun., 16(2), 65-71 (1989).

[20] J. T. Chen, and H.-K. Hong, Review of dual integral representations with emphasis on hypersingularity and divergent series, Invited one-hour Lecture, Proceeding of the Fifth International Colloquium on Numerical Analysis, Plovdiv, Bulgaria, 1996.

[21] J. T. Chen and H.-K. Hong, Boundary Element Method, Second Edition, New World Press, Taipei, Taiwan, 1992(in Chinese).

[22] J. T. Chen and H.-K. Hong, On the dual integral representation of boundary value problem in Laplace equation, Boundary Element Abstracts, 3, 114-116 (1993).

[23] N. M. Günter, Potential theory and its applications to basic problems of mathematical physics, Frederick Ungar Publishing, Co., N.Y., 1967. 
[24] H. F. Buecker, Field singularities and related integral representations, Mechanics of Fracture, Vol.1, G. C. Sih, ed., 1973.

[25] M. Tanaka, V. Sladek and J. Sladek, Regularization techniques applied to boundary element methods, Appl. Mech. Rev., 47(10), 457-499 (1994).

[26] J. T. Chen and F. C. Wong, Analytical derivations for one-dimensional eigenproblems using dual BEM and MRM, Engineering Analysis with Boundary Elements, 20, 25-33 (1997).

[27] A. J. Burton and G. F. Miller, The application of integral equation methods to numerical solution of some exterior boundary value problems, Proc. $R$. Soc. London Ser A, 323, 201-210 (1971).

[28] W. Yeih, J. T. Chen, K. H. Chen and F. C. Wong, A study on the multiple reciprocity method and complex-valued formulation for the Helmholtz equation, Advances in Engineering Softwares, Accepted, 1998.

[29] F. C. Wong, Analysis and experiment for acoustic modes of a cavity containing an incomplete partition, Master thesis, Department of Harbor and River Engineering, National Taiwan Ocean University, Keelung, Taiwan, 1997.

[30] K. H. Chen, Applications of dual integral equations to acoustic problems, Master thesis, Department of Harbor and River Engineering, National Taiwan Ocean University, Keelung, Taiwan, 1997. (in Chinese)

[31] J. T. Chen and K. H. Chen, Dual integral formulation for determining the acoustic modes of a two-dimensional cavity with a degenerate boundary, Engineering Analysis with Boundary Elements, Accepted, 1998.

[32] K. H. Chen, J. T. Chen and D. Y. Liou, Dual boundary element analysis for an acoustic cavity with an incomplete partition, The Chinese Journal of Mechanics, Accepted, 1998.(in Chinese)

[33] M. Petyt, J. Lea J and G. H. Koopmann, The acoustic modes of a rectangular cavity containing a rigid, incomplete partition, J. Sound and Vibration, 53, 71-82 (1976).

[34] M. Petyt, G. H. Koopmann and R. J. Pinnington, A finite element method for determining the acoustic modes of irregular shaped cavities, J. Sound and Vibration, 45, 495-502 (1997).

[35] N. Kamiya, E. Ando and K. Nogae, A new complex-valued formulation and eigenvalue analysis of the Helmholtz equation by boundary element method, Advances in Engineering Softwares, 26, 219-227 (1996). 
[36] A. J. Nowak and A. C. Neves eds., The Multiple Reciprocity Boundary Element Method, Comp. Publ., Southampton, 1994.

[37] C. M. Chang, J. T. Chen and W. D. Yeih, Applications of dual MRM for determining the natural frequencies and natural modes, Proceeding of the 21th National Conference of Theoretical and Applied Mechanics, pp.31-38, Taipei, Taiwan, 1997. 
Table 1 . The former five acoustic frequencies $(\mathrm{Hz})$ using different methods

\begin{tabular}{|c|c|c|c|c|c|}
\hline & mode 1 & mode 2 & mode 3 & mode 4 & mode 5 \\
\hline $\begin{array}{c}\text { Dual } \\
\text { complex-valued } \\
\text { BEM }\end{array}$ & 584 & 1439 & 1518 & 1537 & 1818 \\
\hline $\begin{array}{c}\text { Dual } \\
\text { real-valued } \\
\text { MRM }\end{array}$ & 577 & 1444 & 1529 & 1534 & 1991 \\
\hline $\begin{array}{c}\text { Real part } \\
\text { of dual } \\
\text { BEM }\end{array}$ & 588 & 1444 & 1518 & 1537 & 1827 \\
\hline $\begin{array}{c}\text { Complete } \\
\text { complex-valued } \\
\text { MRM }\end{array}$ & 576 & 1447 & 1510 & 1521 & 1800 \\
\hline $\begin{array}{c}\text { ABAQUS } \\
\text { (AC2D4) }\end{array}$ & 618 & 1421 & 1496 & 1527 & 1780 \\
\hline $\begin{array}{c}\text { ABAQUS } \\
\text { (AC2D8) }\end{array}$ & 605 & 1458 & 1536 & 1563 & 1851 \\
\hline $\begin{array}{c}\text { FEM by } \\
\text { Petyt }\end{array}$ & 591 & 1478 & 1540 & 1570 & 1861 \\
\hline $\begin{array}{c}\text { Measurement } \\
\text { by Petyt }\end{array}$ & 570 & 1470 & 1534 & 1555 & 1840 \\
\hline
\end{tabular}




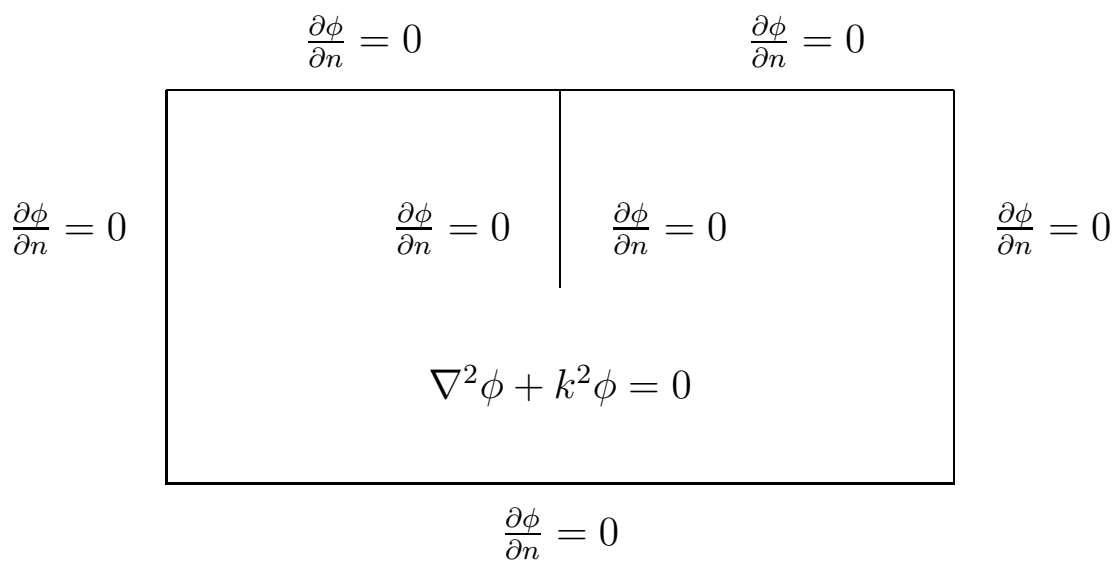

Figure 1. A cavity with an incomplete partition 